\title{
EXOFTALMOS MALIGNO EN HIPERTIROIDISMO CON CRISIS HIPOTIROIDEA
}

\section{MALIGNANT EXOPHTALMOS IN HYPERTHYROIDISM WITH A HYPOTHYROID CRISIS}

\author{
CAMPOS-PAVÓN J ${ }^{1}$, MENCÍA-GUTIÉRREZ E ${ }^{1}$, GUTIÉRREZ-DÍAZ E ${ }^{1}$
}

\begin{abstract}
RESUMEN
Caso clínico: Presentamos un paciente varón de 56 años de edad con un exoftalmos maligno en el contexto de una enfermedad de Graves, que sufrió un claro empeoramiento coincidiendo con su entrada en crisis hipotiroidea, con una evolución tórpida, que requirió de distintas medidas terapéuticas para la reducción de la proptosis.

Discusión: En el manejo de este tipo de pacientes hay que tener en cuenta si cumplen o no criterios de gravedad y/o actividad para aplicar las distintas opciones terapéuticas en el momento preciso.

Palabras clave: Exoftalmos maligno, enfermedad de Graves, oftalmopatía distiroidea, crisis hipotiroidea.
\end{abstract}

\begin{abstract}
Case report: We present the case of a 56-year-old male with a malignant exophthalmos in the context of Graves' disease. He suffered a significant worsening coincident with a hypothyroid crisis, and various therapeutic approaches were required for reduction of the proptosis.

Discussion: When managing this condition, both the severity of the exophthalmos and the activity of the thyroid must be considered when choosing which of the different therapeutic approaches should be employed (Arch Soc Esp Oftalmol 2006; 81: 721-724).
\end{abstract}

Key words: Malignant exophthalmos, Graves' disease, Dysthyroid ophthalmopathy, Hypothyroid crisis.

\section{INTRODUCCIÓN}

Hablar de oftalmopatía distiroidea (OD) equivale a hablar de hipertiroidismo y enfermedad de Graves (EG), aunque un $20 \%$ de los pacientes se presentan en el contexto de un eutiroidismo (1). La prevalencia de la EG ronda el $2 \%$ de la población (2), y el $50 \%$ de los pacientes hipertiroideos con EG presen- tan una oftalmopatía clínicamente significativa, llegando hasta el $90 \%$ si se realiza una tomografía axial computerizada (TAC). Sin embargo, menos del 5\% de estos pacientes presentan una neuropatía óptica (3). La edad de presentación se reparte entre un pico mayoritario entre los 40 y 45 años y otro menor entre los 60 y 69 años. En cuanto a la prevalencia por sexos, predomina el femenino (2-5:1),

Recibido: 18/10/05. Aceptado: 13/12/06.

Servicio de Oftalmología. Hospital 12 de Octubre. Universidad Complutense. Madrid. España.

1 Licenciado en Medicina.

Correspondencia:

E. Mencía-Gutiérrez

C/. Cedro, 23

28250 Torrelodones (Madrid)

España

E-mail: emencia.hdoc@salud.madrid.org 
dato que contrasta con la prevalencia de la propia enfermedad per se (5-10:1) (4).

\section{CASO CLÍNICO}

Varón de 56 años de edad con antecedentes de neoplasia de laringe, diabetes mellitus tipo 2 , exfumador y ex-bebedor, diagnosticado de crisis hipertiroidea. En ese momento presentaba un exoftalmos de 23 y $25 \mathrm{~mm}$ sin limitación de los movimientos oculares (fig. 1), agudeza visual de 0,7 con su corrección en ambos ojos (AO), fondo de ojo con retinopatía diabética leve y engrosamiento de los músculos rectos internos en la TAC orbitario.

Un mes más tarde presentó retracción de ambos párpados con limitación de la motilidad superior y lateral en AO. La tensión ocular (TO) era de 26 $\mathrm{mmHg}$ en ojo derecho y $25 \mathrm{mmHg}$ en ojo izquierdo. Se instauró tratamiento con beta-bloqueante tópico y tratamiento corticoideo sistémico con 1 $\mathrm{gr} / 24 \mathrm{~h}$ de metil-prednisolona iv 3 pulsos.

El exoftalmos y la TO fueron aumentando, por lo que se añadieron inhibidores de la anhidrasa carbónica tópicos, y se derivó al paciente para radioterapia orbitaria con $2.000 \mathrm{~Gy}$.

La evolución fue desfavorable; la proptosis aumentó a 27 y $30 \mathrm{~mm}$ y la TAC confirmó el aumento del engrosamiento de los músculos rectos internos (fig. 2). Se produjo oftalmoplejía en $\mathrm{AO}$ y exposición corneal en ojo izquierdo (fig. 3) por lo que se realizó descompresión bilateral orbitaria con fractura de las paredes inferior, interna y externa

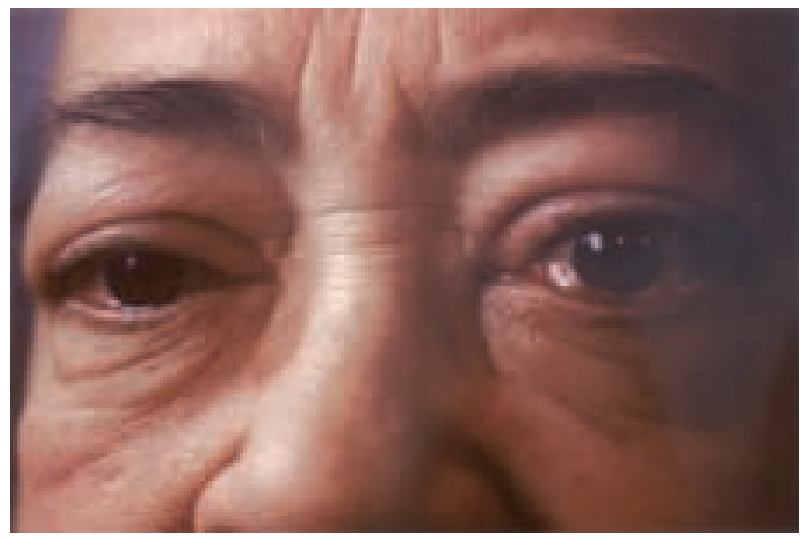

Fig. 1: Un año de historia de proptosis, diplopía, conjuntivitis crónica, y dolor de cabeza constante. La medida de la exoftalmometría tipo Hertel fue: OI $27 \mathrm{~mm}$; OD $30 \mathrm{~mm}$.

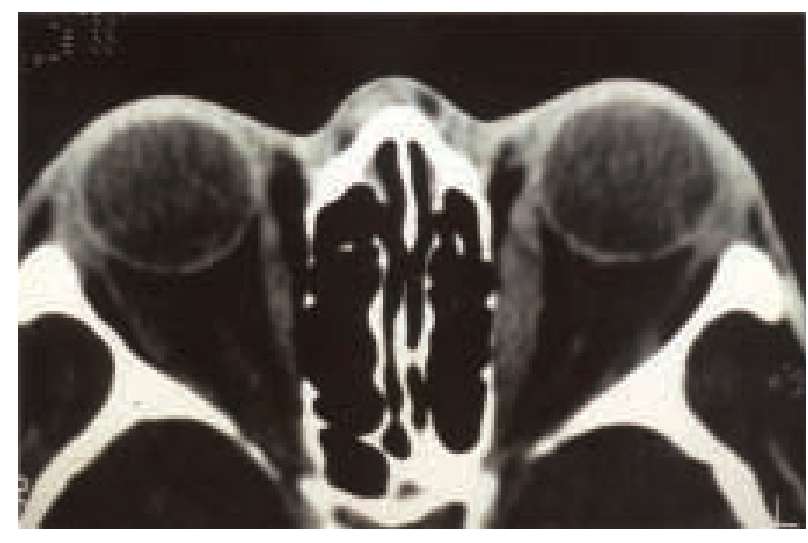

Fig. 2: La TAC muestra un incremento de tamaño de los músculos rectos, con aumento de la grasa intraconal. Se aprecia una severa elongación de los nervios ópticos.

(fig. 4). El control hormonal reveló una severa crisis hipotiroidea (TSH 44,5 uUI/mL $(0,5-5,0)$, T3 42 ng/dL (60-180), T4 Total 02,4 ng/dL (5,0-12,0), T4 Libre $0,3 \mathrm{ng} / \mathrm{dL}(0,7-1,8)$. El exoftalmos siguió empeorando, ante lo cual se realizó una lipectomía en ambas órbitas ( 1 cc de grasa), que muestra músculos y tejidos orbitarios infiltrados, enucleación del ojo izquierdo por glaucoma absoluto, y tarsorrafia externa e interna en ojo derecho . Posteriormente, las hormonas tiroideas se normalizaron (TSH 14,3, T4 Total 5,9 y T4 Libre 1,1), la proptosis disminuyó de forma manifiesta y la motilidad ocular aumentó, abriéndose las tarsorrafias. La exploración de OD mostró AV de 0,1 como consecuencia de la neuropatía óptica severa, TO de $18 \mathrm{mmHg}$, y

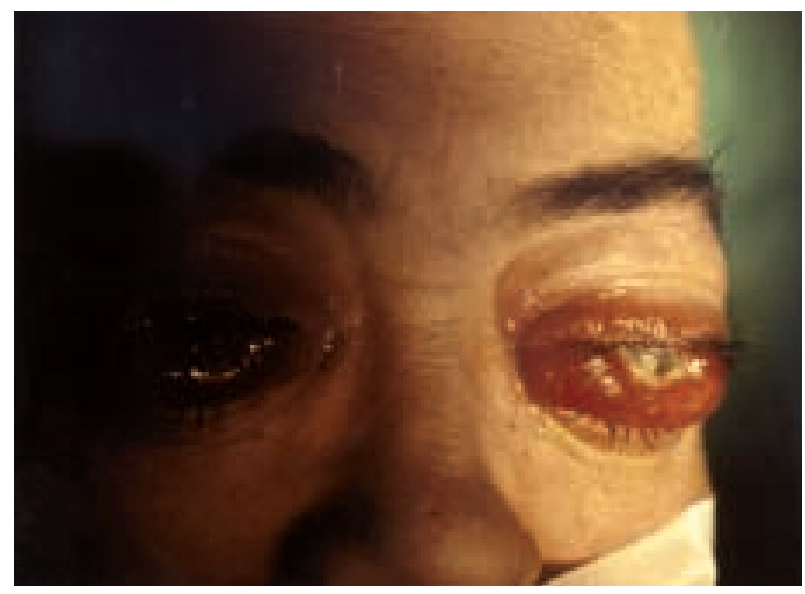

Fig. 3: Aspecto clínico preoperatorio con proptosis extrema, exposición corneal, gran quemosis y oftalmoplejía completa. 


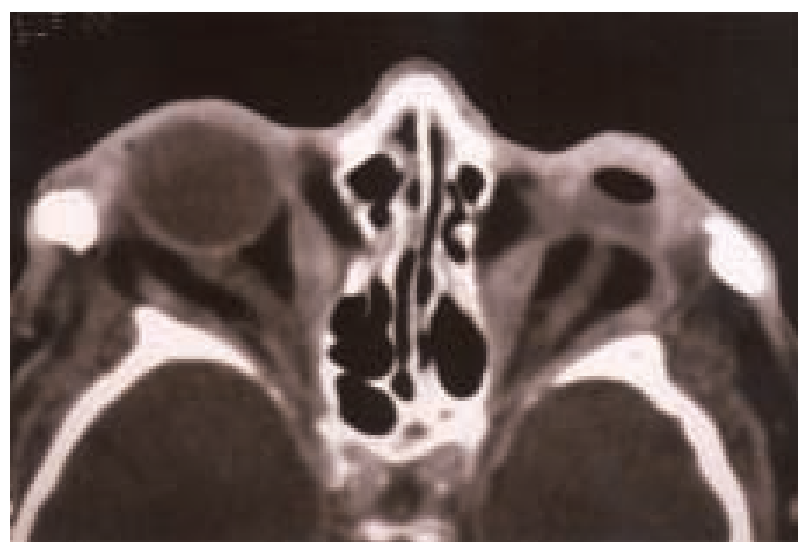

Fig. 4: TAC postoperatoria donde se observa fractura ósea para la descompresión, de las paredes orbitaria internas y laterales, con una mejor distribución de los contenidos orbitarios. OI enucleado.

retinopatía diabética leve-moderada. El paciente permanece estable después de 9 años de evolución (fig. 5).

\section{DISCUSIÓN}

La patogenia de la EG está condicionada por la aparición de autoanticuerpos estimulantes del receptor TSH del tiroides. Por tanto, no constituye solamente una hiperactividad glandular responsable del aumento de la producción de hormonas tiroideas, sino que además tiene un claro componente inflamatorio-autoinmune, confirmado por la presencia de infiltración de linfocitos $\mathrm{T}$ y $\mathrm{B}$ en las biopsias de tejido tiroideo en pacientes con EG.

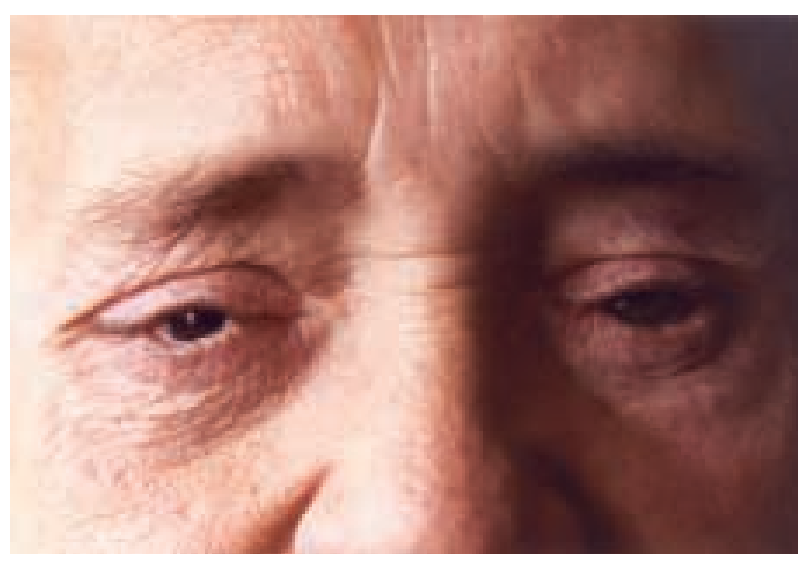

Fig. 5: Después de 9 años de evolución el paciente no muestra proptosis ni signos de actividad mixomatosa.
La patogenia de la OD es más polémica. Existe cierto grado de hiperactividad simpática, pero mediante las nuevas técnicas de procesamiento genético se han logrado aislar receptores de TSH en los fibroblastos y grasa orbitaria iguales a los tiroideos. Estos constituirían un reclamo para las células del sistema inmune que infiltrarían los tejidos retrorbitarios y serían responsable de la síntesis por los fibroblastos orbitarios de mucopolisacáridos (sustancias muy hidrófilas que provocan el aumento de volumen) y colágeno (responsable de la fibrosis y retracción de los músculos en la fase inactiva de la enfermedad).

La OD requiere un abordaje multidisciplinar. Debe tenerse en cuenta si cumple o no criterios de gravedad y/o actividad (tabla I) para aplicar las distintas opciones terapéuticas en el momento preciso y evitar la yatrogenia. En la fase activa, las medidas consideradas más eficaces y recomendadas son los corticoides a dosis altas, la radioterapia orbitaria y la descompresión quirúrgica. Otros tratamientos con resultados esperanzadores son los análogos de la somatoestatina y algunos inmunosupresores como la ciclosporina o el metrotexate. Si no hay actividad, solo está indicada cirugía de la musculatura extraocular para corregir la diplopia y la realización de tarso y blefarorrafias para prevenir la queratitis por exposición. En caso de que la OD cumpla criterios de gravedad, la decisión de realizar una descompresión quirúrgica o médica depende de la experiencia, aunque si existe neuropatía compresiva tiende a preferirse la cirugía (5).

Es necesario realizar estudios para confirmar los resultados de los análogos de la somatostatina, ya que estos tienen menos contraindicaciones y efectos secundarios que los corticoides, así como los factores de riesgo de la EG y de la OD, estando demostrado que el abandono del tabaquismo mejora la situación y el pronóstico de la enfermedad y aumenta la efectividad de las distintas medidas terapéuticas.

La edad y el sexo, así como los antecedentes de tabaquismo, radioterapia y diabetes mellitus, son a tener en cuenta en pacientes con hipertiroidismo, ya que además si se presenta una crisis hipotiroidea, el

Tabla I. Criterios de severidad de la oftalmopatía distiroidea. Bartelena $L$ et al 2002 (5)

\begin{tabular}{llll}
\hline Grado & Proptosis & Diplopía & Neuropatía \\
Leve & $19-20 \mathrm{~mm}$ & Intermitente & Subclínica \\
Moderado & $21-23 \mathrm{~mm}$ & Inconstante & AV: 0,8-0,5 \\
Severo & $>23 \mathrm{~mm}$ & Constante & AV: $<0,5$ \\
\hline \hline
\end{tabular}


resultado puede ser un exoftalmos maligno con graves repercusiones funcionales y estéticas oculares.

\section{BIBLIOGRAFÍA}

1. Clauser L, Galie M, Sarti E, Dallera V. Rationale of treatment in Graves ophthalmopathy. Plast Reconstr Surg 2001; 108: 1880-1894.

2. Dolman PJ, Rootman J. VISA classification for Graves orbitopathy. Ophthal Plast Reconstr Surg 2006; 22: 319-324.
3. Ben Simon GJ, Syed M, Douglas R, Schwarz R, Goldberg $R A$, McCann JD. Clinical manifestations and treatment outcome of optic neuropathy in thyroid-related orbitopathy. Ophthalmic Surg Lasers Imaging 2006; 37: 284290.

4. Bartley GB. The epidemiologic characteristics and clinical course of ophthalmopathy associated with autoimmune thyroid disease in Olmsted County, Minnesota. Trans Am Ophthalmol Soc 1994; 92: 477-588.

5. Bartelena L, Marcocci C, Tanda ML, Pinchera A. Management of thyroid eye disease. Eur J Nucl Med 2002; 29: S458-S465. 